\title{
Providing expert guidance in the evidence-based medicine era?
}

\author{
Bram Rochwerg, MD, MSc $\mathbb{D}$
}

Received: 26 November 2018/Revised: 27 November 2018/Accepted: 28 November 2018/Published online: 3 January 2019

(c) Canadian Anesthesiologists' Society 2019

Thousands of Canadians with end-organ dysfunction wait on recipient lists hoping to receive an organ before they die from their underlying disease. ${ }^{1}$ In this issue of the Journal, Zavalkoff et al. provide expert guidance regarding the identification of potential organ donors and methods for ensuring system accountability. ${ }^{2}$ Their guidance is based on a consensus conference of experts in the field of organ donation and other stakeholders from across Canada, including patient and family partners. Themes addressed include: 1) expectations of donors, recipients, and their families, 2) donor identification and referral, 3) enhancing accountability-identifying gaps and solutions, and 4) enhancing accountability-improving quality and safety. While it is clearly a priority to increase the number of available organs for transplantation, of equal importance are the procedures addressing the identification of potential donors and those assessing system accountability, which are fragmented and lack inter-provincial standardization. The objective of this document is to fill this void and address these current system-based limitations.

How does a group go about generating optimal guidance on a topic such as this? In the evidence-based medicine era, we strive to provide hard data to support all suggestions for clinical care. ${ }^{3}$ The Grading of Recommendations, Assessment, Development, and Evaluations (GRADE) framework is one specific clinical practice guideline methodology that ensures clear linkage between

B. Rochwerg, MD, MSc ( $\square)$

Department of Medicine (Division of Critical Care), McMaster University, Hamilton, ON, Canada

e-mail: rochwerg@mcmaster.ca

Department of Health Research Methods, Evidence and Impact, McMaster University, Hamilton, ON, Canada recommendations and the evidence that informs them. ${ }^{4}$ Using GRADE, questions of interest are formatted using PICO (patient, intervention, comparator, outcome). Evidence is then summarized using comprehensive systematic reviews, and final recommendations are generated incorporating stakeholder input and considering domains such as the perceived balance between benefits/ harms, cost, acceptability, feasibility, and impact on health equity. ${ }^{5,6}$

This group did not use GRADE or a formal guideline development methodology. Despite its strengths, not every clinical question can be addressed using this comprehensive guideline development process. This is especially true for health system-based, quality improvement, and communication documents. Nevertheless, the guidance provided by Zavalkoff et al. is certainly trustworthy and helpful, and should be adopted by donor management programs across Canada in order to improve donor identification processes. A number of features lend credibility to their effort. For one, the panel was composed of a diverse group of stakeholders including non-physicians, ethicists, and patient/family partners; this is crucial to ensure all viewpoints are considered.

Rather than performing systematic reviews with certainty assessments (as would be expected with GRADE), narrative evidence summaries were provided to participants with the goal of guiding discussion. This represents a significant departure from formal guideline development and the limitations of this approach need to be carefully considered. If one were offering practice recommendations regarding the use of a new drug for a certain medical condition, it should be absolutely mandatory that these recommendations be based on the most up-to-date systematic review and meta-analysis of available randomized controlled trial data. This approach 
clearly becomes more difficult when addressing topics such as organ donation. You can imagine how summarizing the literature examining whether and when healthcare providers should address organ donation as part of end-of-life care may be challenging. Good practice statements, a creation by the GRADE working group, may be one potential solution. These statements, however, have limitations, and consensus on when they are most applicable is still uncertain. ${ }^{7,8}$ The alternative-providing expert consensus opinion without any attempt to summarize the relevant literature-may appear paternalistic. The optimal approach in these situations remains unclear. Nevertheless, the authors have done their best to incorporate data into their guidance, without dedicating (potentially) wasted resources to a multitude of unhelpful systematic reviews. Understanding this difference between formal recommendations (subjected to guideline methodology) and more expert-driven consensus statements is crucial and often underappreciated by readers and end-users. The authors have done an excellent job in being transparent and clear in the process they employed. It is for these reasons they have avoided terminology such as "recommendations" and "clinical practice guideline", to clearly differentiate their approach from the more formal guideline development process.

The document provides important considerations around ethical and legal aspects of organ donation. These topics are often avoided in similar reports due to differences in local jurisdiction, conflicting laws, lack of expertise, or with the goal of limiting contention. By providing context to the Canadian environment, and by addressing these considerations in a harmonious manner including expert legal and ethical input, Zavalkoff $e t$ al. have enhanced the uptake of their guidance immensely.

At least in clinical medicine, no guidance document is absolute, and no guidance is to be applied dogmatically and to every situation. Every recommendation, suggestion, or expert statement must be evaluated in the local context, discussed between relevant end-users (including, but not limited to, patients, families, clinicians, and administrators) and applied in a way that makes the most sense to all involved. In this light, expert guidance such as this serves an important role. Without a doubt, this document will lead to improvements in the organ donation process. For centres experienced with organ donation, it will ensure standardization and act as a reference point for current practice. For centres with less experience, it will provide benchmarks or targets to improve donor identification processes. As research in organ donation practice continues, a more expansive evidence-base may lend itself to formal guideline development. In the meantime, clinicians and donation programs around the country should read this guidance document closely and work to improve their donor identification and management systems, with the ultimate goal of providing more highquality organs for Canadians who are desperately in need.

\section{Fournir des conseils d'expert à l'ère de la médecine fondée sur des données probantes?}

Des milliers de Canadiens souffrant d'une atteinte terminale d'un organe sont sur des listes d'attente dans l'espoir de bénéficier d'une transplantation avant de mourir de leur maladie sous-jacente. ${ }^{1}$ Dans ce numéro du Journal, Zavalkoff et coll. proposent un ensemble de conseils d'expert concernant l'identification de donneurs d'organes potentiels et des méthodes permettant d'assurer la responsabilisation du système. ${ }^{2}$ Leur opinion repose sur une conférence de consensus d'experts dans le domaine des dons d'organes et d'autres acteurs venant de tout le Canada, y compris des patients et leurs familles. Ils ont fait un bon travail en constituant un panel multidisciplinaire et varié. Les thèmes abordés étaient notamment : 1) les attentes des donneurs, des receveurs et de leurs familles, 2) l'identification et l'orientation des donneurs, 3) l'amélioration de la responsabilité concernant l'identification des lacunes et leurs solutions, et 4) l'amélioration de la responsabilité avec l'amélioration de la qualité et de la sécurité. Bien que l'augmentation du nombre d'organes à transplanter disponibles soit une priorité indiscutable, les procédures concernant l'identification des donneurs potentiels et celles évaluant la responsabilisation du système sont tout aussi importantes. Or, ces dernières sont fragmentées et il y a un manque de normalisation interprovinciale. L'objectif de ce document est de combler ce vide et d'aborder ces limites actuelles liées au système.

Comment un groupe peut-il progresser et générer des conseils optimaux sur un tel sujet? À l'ère de la médecine fondée sur des données probantes, nous nous efforçons de fournir des données solides pour évaluer toutes les propositions de soins cliniques. ${ }^{3}$ Le cadre GRADE (Classification des recommandations, évaluations et développement [Grading of Recommendations, Assessment, Development, and Evaluations]) est une méthodologie spécifique de ligne directrice de la pratique clinique qui assure une liaison claire entre les recommandations et les données probantes qui les alimentent. $^{4}$ Avec GRADE, des questions d'intérêt peuvent être formatées en utilisant la procédure PICO (Patient, Intervention, Comparateur, résultat [Outcome]). Les données probantes sont alors synthétisées dans des 
analyses systématiques complètes et des recommandations finales sont générées en incorporant l'avis des parties prenantes et en prenant en compte des domaines tels que l'équilibre perçu entre les avantages et les effets nuisibles, le coût, l'acceptabilité, la faisabilité et impact sur l'équité des soins. ${ }^{5,6}$

Ce groupe n'a pas utilisé GRADE ou une méthodologie formelle d'élaboration de lignes directrices. En dépit de ses points forts, toutes les questions cliniques ne peuvent pas être abordées au moyen de la procédure globale d'élaboration des lignes directrices. Cela est particulièrement vrai pour les documents concernant les communications, l'amélioration de la qualité et basés sur le système de santé. Néanmoins, la guidance procurée par Zavalkoff et coll. est certainement fiable et utile; elle devrait être adoptée par les programmes de gestion des donneurs dans tout le Canada pour améliorer les processus d'identification des donneurs. Plusieurs caractéristiques, en particulier, rendent leur effort crédible, notamment la diversité des parties prenantes dont des non-médecins, des éthiciens et des partenaires patients/familles; cela est crucial pour garantir la prise en compte de tous les points de vue.

Plutôt que de réaliser des études systématiques avec évaluations de certitude (comme on s'y attendrait avec GRADE), le panel a plutôt fourni aux participants des synthèses narratives des données probantes dans le but de guider le débat. Cela représente un écart significatif par rapport à l'élaboration formelle de lignes directrices et les limites de cette démarche doivent être soigneusement analysées. Si quelqu'un proposait des recommandations aux praticiens pour l'utilisation d'un nouveau médicament contre une maladie spécifique, il serait absolument obligatoire de baser ces recommandations sur les plus récentes analyses systématiques et méta-analyses des essais comparatifs randomisés. Cette démarche est manifestement plus difficile à suivre quand il s'agit d'un sujet tel que le don d'organes. Vous pouvez imaginer combien cela est difficile de résumer les publications sur les questions de savoir si et quand les prestataires de santé doivent aborder la question des dons d'organes dans le cadre des soins de fin de vie. Les énoncés de bonnes pratiques, une création de groupe de travail GRADE, pourraient représenter une solution. Néanmoins, ces énoncés ont des limites et savoir quand elles sont le plus applicables ne fait pas encore l'objet d'un consensus définitif. ${ }^{7,8}$ L'autre possibilité (c'est-à-dire fournir l'avis d'un consensus d'experts sans aucune tentative de synthèse de la documentation) pourrait sembler paternaliste. La démarche optimale à adopter dans ces situations reste floue. Les auteurs ont néanmoins fait de leur mieux pour incorporer des données dans leur avis, sans gaspiller (potentiellement) des ressources consacrées à une multitude d'analyses systématiques qui n'apporteraient rien. Comprendre cette différence entre des recommandations formelles (soumises à la méthodologie des lignes directrices) et des énoncés de consensus davantage menés par des experts est crucial et souvent insuffisamment saisi par les lecteurs et utilisateurs finaux. Les auteurs ont fait un excellent travail: ils ont exposé de façon claire et transparente le processus qu'ils ont suivi. C'est pour cette raison qu'ils ont évité d'employer une terminologie telle que «recommandations» et «lignes directrices de pratique clinique» pour clairement différencier leur démarche d'un processus d'élaboration plus formel de lignes directrices.

Ce document propose d'importantes réflexions sur les aspects éthiques et légaux du don d'organes. Ces thèmes sont souvent évités dans les rapports similaires en raison des différences liées aux juridictions locales, aux lois contradictoires, à l'absence d'expertise ou dans le but d'éviter les conflits. En fournissant un contexte propre à l'environnement canadien et en abordant ces questions de manière harmonieuse, incluant un avis d'expertise légale et éthique, Zavalkoff et coll. ont considérablement renforcé la portée de leurs conseils.

En médecine clinique, au moins, aucun document d'orientation n'a de valeur absolue et aucun avis ne doit être appliqué de manière dogmatique et dans tous les cas. Chaque recommandation, suggestion ou affirmation d'expert doit être évaluée dans le contexte local, discutée entre utilisateurs pertinents (y compris, mais sans s'y limiter, les patients, les familles, les cliniciens et les administrateurs) et appliquée d'une manière qui ait le plus de sens pour toutes les parties concernées. Dans ce sens, les conseils d'expert tels que ceux-ci jouent un rôle important. Ce document entraînera, sans aucun doute, des améliorations au processus de dons d'organes. Pour les centres ayant l'expérience du don d'organes, il assurera une standardisation et agira comme référence pour les pratiques actuelles. Pour ceux qui sont moins expérimentés, il fournira des repères ou des cibles pour améliorer les processus de repérage de donneurs. Avec la poursuite des pratiques de dons d'organe, une base de données probantes plus large pourrait déboucher d'elle-même sur l'élaboration de lignes directrices officielles. En attendant, les cliniciens et programmes de dons d'organe $\mathrm{du}$ pays doivent lire soigneusement ce document d'orientation et travailler à l'amélioration de l'identification de leurs donneurs et des systèmes de gestion, dans le but ultime de fournir davantage d'organes à des Canadiens qui en ont désespérément besoin. 
Conflicts of interest None declared.

Editorial responsibility This submission was handled by Dr. Sangeeta Mehta, Associate Editor, Canadian Journal of Anesthesia.

Responsabilité éditoriale Cet article a été traité par la Dre Sangeeta Mehta, rédactrice adjointe, Journal canadien d'anesthésie.

Conflits d'intérêts Aucun déclaré.

\section{References}

1. Canadian Institute for Health Information. e-Statistics on organ transplants, waiting lists and donors. Secondary e-Statistics on organ transplants, waiting lists and donors 2018. Available from URL: https://www.cihi.ca/en/e-statistics-on-organ-transplantswaiting-lists-and-donors (accessed November 2018).

2. Zavalkoff S, Shemie SD, Grimshaw JM, et al. Potential organ donor identification and system accountability: expert guidance from a Canadian consensus conference. Can J Anesth 2019; 66: DOI: https://doi.org/10.1007/s12630-018-1252-6.
3. Institute of Medicine. Clinical Practice Guidelines We Can Trust. Washington DC: National Academies Press (US); 2011.

4. Guyatt GH, Oxman AD, Vist GE, et al. GRADE: an emerging consensus on rating quality of evidence and strength of recommendations. BMJ 2008; 336: 924-6.

5. Guyatt GH, Oxman AD, Kunz R, et al. GRADE guidelines: 2. Framing the question and deciding on important outcomes. J Clin Epidemiol 2011; 64: 395-400.

6. Guyatt GH, Oxman AD, Kunz, R, et al. Going from evidence to recommendations. BMJ 2008; 336: 1049-51.

7. Weiss MJ, Hornby L, Shemie SD, Appleby A, Rochwerg $B$. GRADEing the un-GRADE-able: a description of challenges in applying GRADE methods to the ethical and implementation questions of pediatric organ donation guidelines. J Clin Epidemiol 2018; 103: 134-7.

8. Guyatt GH, Schünemann HJ, Djulbegovic B, et al. Guideline panels should not GRADE good practice statements. J Clin Epidemiol 2014; 68: 597-600.

Publisher's Note Springer Nature remains neutral with regard to jurisdictional claims in published maps and institutional affiliations. 